\title{
Synthesis of gold nanoparticles using renewable Punica granatum juice and study of its catalytic activity
}

\author{
Shib Shankar Dash • Braja Gopal Bag
}

Received: 10 September 2012/ Accepted: 22 November 2012/Published online: 8 December 2012

(C) The Author(s) 2012. This article is published with open access at Springerlink.com

\begin{abstract}
Punica granatum juice, a delicious multivitamin drink of great medicinal significance, is rich in different types of phytochemicals, such as terpenoids, alkaloids, sterols, polyphenols, sugars, fatty acids, aromatic compounds, amino acids, tocopherols, etc. We have demonstrated the use of the juice for the synthesis of gold nanoparticles (AuNPs) at room temperature under very mild conditions. The synthesis of the AuNPs was complete in few minutes and no extra stabilizing or capping agents were necessary. The size of the nanoparticles could be controlled by varying the concentration of the fruit extract. The AuNPs were characterized by surface plasmon resonance spectroscopy, high resolution transmission electron microscopy, fourier transform infrared spectroscopy and $\mathrm{X}$-ray diffraction studies. Catalytic activity of the synthesized colloidal AuNPs has also been demonstrated.
\end{abstract}

Keywords Gold nanoparticle - Green synthesis - Punica granatum $\cdot$ Catalytic reduction

\section{Introduction}

Gold nanoparticles (AuNPs) in the size range of 1-100 nm exhibit unique optical, electronic and catalytic properties compared to the bulk solids and are biocompatible and non-toxic (Jain et al. 2008). During the past two decades an

Electronic supplementary material The online version of this article (doi:10.1007/s13204-012-0179-4) contains supplementary material, which is available to authorized users.

S. S. Dash · B. G. Bag ( $\bowtie)$

Department of Chemistry and Chemical Technology, Vidyasagar University, Midnapore 721 102, West Bengal, India

e-mail: braja@mail.vidyasagar.ac.in enormous effort has been invested in the investigations of AuNPs because of its applications in catalysis (Zhang et al. 2012), biotechnology (Murphy et al. 2008), drug delivery, biodiagnostics (Dykman and Khlebtsov 2012; Beeram and Zamborini 2010; Saha et al. 2012), etc. AuNPs exhibit different colors depending upon their size, shape and degree of aggregation. Though the use of colloidal gold particles can be traced back to 5th to 4th century B.C., the scientific method for the reductive synthesis of colloidal gold can be traced back to 1857, when Michael Faraday reported a reductive method for the preparation of gold hydrosols from an aqueous solution of chloroaurate using phosphorus dissolved in carbon disulfide (Daniel and Astruc 2004; Faraday 1857). Synthesis of metal nanoparticles using plant extracts containing amino acids, organic acids, polyphenols, vitamins, polysaccharides, etc. has received tremendous attention in recent years because of the renewable nature of plant metabolites (Anastas and Kirchhoff 2002; Aromal et al. 2012). The reduction of the metal salts usually take place in water under mild reaction conditions where the plant extract acts both as reducing as well as stabilizing agent. The extracts of Bayberry tannin (Huang et al. 2010), Alfa-alfa (Montes et al. 2011), Aloe vera (Chandran et al. 2006), Terminalia arjuna bark (Majumdar and Bag 2012), Azadirachta indica leaf (Shiv Shankar et al. 2004), Syzygium cumini (Kumari et al. 2010), etc. have been utilized for the synthesis of AuNPs. During the course of our investigations on the utilization of plant secondary metabolites as renewables (Bag and Dash 2011; Bag et al. 2012; Bag and Paul 2012; Bag and Majumdar 2012), it occurred to us that the medicinally important fruit extract of Punica granatum, rich in different types of plant secondary metabolites including polyphenolic compounds, can be utilized for the synthesis of AuNPs from $\mathrm{HAuCl}_{4}$. Herein we report a very mild and 
environmentally friendly method for the synthesis of AuNPs from the fruit extract of $P$. granatum without any additional capping or stabilizing agents. The AuNPs were characterized by surface plasmon resonance spectroscopy, high resolution transmission electron microscopy (HRTEM), X-ray diffraction (XRD) and fourier transform infrared spectroscopy (FTIR). The synthesized colloidal AuNPs have been used as a catalyst for the sodium borohydride reduction of 4-nitrophenol to 4-aminophenol, and the catalytic rate constant has also been determined.

\section{Experimental}

Synthesis and characterization of nanoparticles

$\mathrm{HAuCl}_{4}$ (36.5 mg) was dissolved in deionized water $(10 \mathrm{~mL})$ to obtain a $10.7 \mathrm{mM} \mathrm{Au}(\mathrm{III})$ stock solution. Aliquots of $\mathrm{Au}(\mathrm{III})$ solution $(0.2 \mathrm{~mL}, 10.7 \mathrm{mM})$ were added drop-wise to the juice extract to prepare a series of stabilized AuNPs, where concentration of the juice extract varied from 480 to $2,400 \mathrm{mg} \mathrm{L}^{-1}$ and the concentration of $\mathrm{Au}$ (III) remained fixed at $0.54 \mathrm{mM}$ (see supporting information for a detailed procedure). UV-vis spectroscopy of the solutions was carried out after $24 \mathrm{~h}$ of mixing $\mathrm{HAuCl}_{4}$ and the purified juice extract, and a band in the vicinity of $530 \mathrm{~nm}$ confirmed the formation of AuNPs. Polydispersive nature and size distribution of AuNPs were confirmed by TECNAI $\mathrm{G}^{2} 20 \mathrm{HRTEM}^{\mathrm{H}}$ an accelerating voltage of $200 \mathrm{kV}$. X-ray diffraction patterns of the stabilized AuNPs were recorded in Rigaku Miniflex II diffractometer with $\mathrm{Cu}-\kappa$ radiation $(\lambda=1.54 \AA$ ). Mass spectra were recorded in Shimadzu GCMS QP 2100 Plus. UV-vis spectra were recorded in Shimadzu 1601 spectrophotometer. FTIR spectra of the samples were recorded using a Lambda Scientific FTIR-7600 instrument with $\mathrm{KBr}$ pellet in transmittance mode.

\section{Results and discussion}

The juice of $P$. granatum contains different classes of organic compounds, such as sugars, organic acids, amino acids, sterols, terpenoids, fatty acids, hydroxybenzoic acids, flavones, anthocyanines, etc. (Prakash and Prakash 2011). The antioxidant flavanoids and anthocyanines provide the $P$. granatum juice a brilliant color. Several compounds having multiple phenolic hydroxyl groups, such as ellagic acid, gallic acid, quinic acid, caffeic acid, $(+)$ catechin, (-) epicatechin, quercetin, rutin, cyanidin, cyanidin3-o-glucoside, delphinidin, delphinidin-3-o-glucoside, etc. are present in the juice extract of $P$. granatum (supporting information Figs. S1 and S2) (Prakash and Prakash 2011).
The $o$-dihydroxy groups can easily form five membered chelate ring and also be oxidized to the corresponding benzoquinones by either air or metal ions. Thus, these phenolic hydroxyl containing compounds can be used as an effective reducing agent for $\mathrm{Au}(\mathrm{III})$ which has a high oxidation-reduction potential (Ferrer et al. 1999). The $\mathrm{Au}(0)$ thus formed may collide with the neighboring $\mathrm{Au}(0)$ atoms forming $\mathrm{Au}$ nanoparticles and get stabilized by the concomitantly generated benzoquinone derivatives (Kim and Nakano 2005). To test this, aliquots of aqueous $\mathrm{HAuCl}_{4}$ solutions $(0.2 \mathrm{~mL}, 10.7 \mathrm{mM})$ were treated with increasing concentrations of the juice extract of $P$. granatum (480-2,400 $\mathrm{mg} \mathrm{L}^{-1}$ ). Interestingly, we observed the appearance of pinkish red coloration instantly indicating the formation of AuNPs. The intensity of the color increased on keeping the solution at room temperature for several hours (Fig. 1).

Due to the charge transfer interactions between the metal and the chloro ligands, a strong absorption peak at $221 \mathrm{~nm}$ and a shoulder peak at $290 \mathrm{~nm}$ were observed in the $\mathrm{UV}$-vis spectrum of $\mathrm{HAuCl}_{4}$. With increasing concentration of the $P$. granatum juice extract, the intensities of these peaks decreased and new peaks in the region of

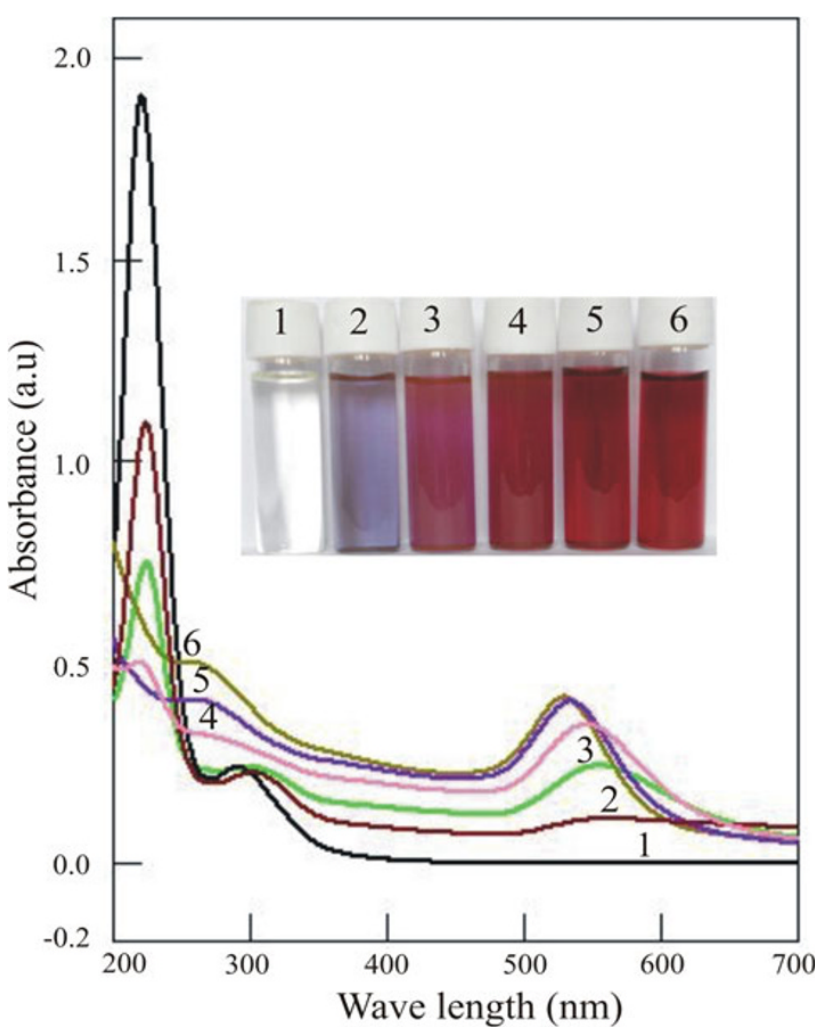

Fig. 1 UV-vis spectra of (1) $\mathrm{HAuCl}_{4}(0.42 \mathrm{mM}),(2-6)$ AuNP's at $480,960,1,440,1,920$, and $2,400 \mathrm{mg} \mathrm{L}^{-1}$ concentrations of $P$. granatum extract, respectively. Inset photograph of the vials containing the solutions (after $24 \mathrm{~h}$ of mixing) 
530-559 $\mathrm{nm}$ appeared due to surface plasmon resonance (SPR) of the AuNPs (Esumi et al. 2000). A blue-shift of the SPR band was observed from 559 to $530 \mathrm{~nm}$ with increasing concentration of the juice extract from 480 to $2,400 \mathrm{mg} \mathrm{L}^{-1}$. This blue-shift is perhaps due to the formation of more stabilized, smaller-sized nanoparticles with increasing concentration of the $P$. granatum juice extract. Above $2,400 \mathrm{mg} \mathrm{L}^{-1}$ concentration of the juice extract, no further increase of the SPR band was observed, perhaps due to complete reduction of the accessible $\mathrm{Au}$ (III) ions to $\mathrm{Au}(0)$ (Huang et al. 2010). A broad peak in the 270-275 nm region observed in stabilized AuNPs might be due to the quinone moiety resulting from the oxidation of phenolic compounds.

The crystalline nature and purity of the metallic face centered cubic AuNPs were confirmed from the five intense peaks in wide angle X-ray diffraction of AuNPs at $2 \theta=38.2^{\circ}, 44.3^{\circ}, 64.7,^{\circ} 77.5^{\circ}$ and $81.7^{\circ}$ which can be indexed as (111), (200), (220), (311) and (222) reflections, respectively (supporting information Fig. S3 for details), based on the comparison with the standard data given by JCPDS file no. 04-0784.

The morphologies of the Au nanoparticles obtained at different concentrations of the $P$. granatum juice extract were characterized by transmission electron microscopy. The size of the nanoparticles formed at various concentrations of the $P$. granatum juice extract is shown in Fig. 2 (supporting information Fig. S4). We observed that the average particle size shows a gradual decrease (from 35.8 to $23.1 \mathrm{~nm}$ ) when concentration varies from 1,440 to 2,400 $\mathrm{mg} \mathrm{L}^{-1}$ (Fig. 2c, e) that is consistent with the blue-shift observed in SPR spectra (Fig. 1). Nanoparticles formed were of different shapes, such as triangular, pentagonal, hexagonal as well as spherical. The gold nano particles are so stable that no further aggregation of the AuNPs takes place on keeping the solution for several months. Selected area electron diffraction (SAED) pattern revealed four rings of Brags reflections corresponding to crystalline fcc nature for AuNPs (Fig. 2f). Compositional analysis of the nanoparticles carried out by energy disperse X-ray (EDX) spectroscopy indicated the presence of biomolecules consisting of carbon and oxygen along with the metallic AuNPs (supporting information Fig. S5).

FTIR spectra of the purified $P$. granatum juice extract and the stabilized AuNPs synthesized from it were compared (supporting information Fig. S6). A peak near $3,383 \mathrm{~cm}^{-1}$ in the spectrum of the extract was broad probably due to intermolecular hydrogen bonding among $-\mathrm{OH}$ or $-\mathrm{OH} /-\mathrm{NH}$ functionality. The peak in the vicinity of $2,931 \mathrm{~cm}^{-1}$ is due to saturated $\mathrm{C}-\mathrm{H}$ stretching vibration. The presence of carboxyl group in the biomass was confirmed from the strong absorption band at $1,732 \mathrm{~cm}^{-1}$. The
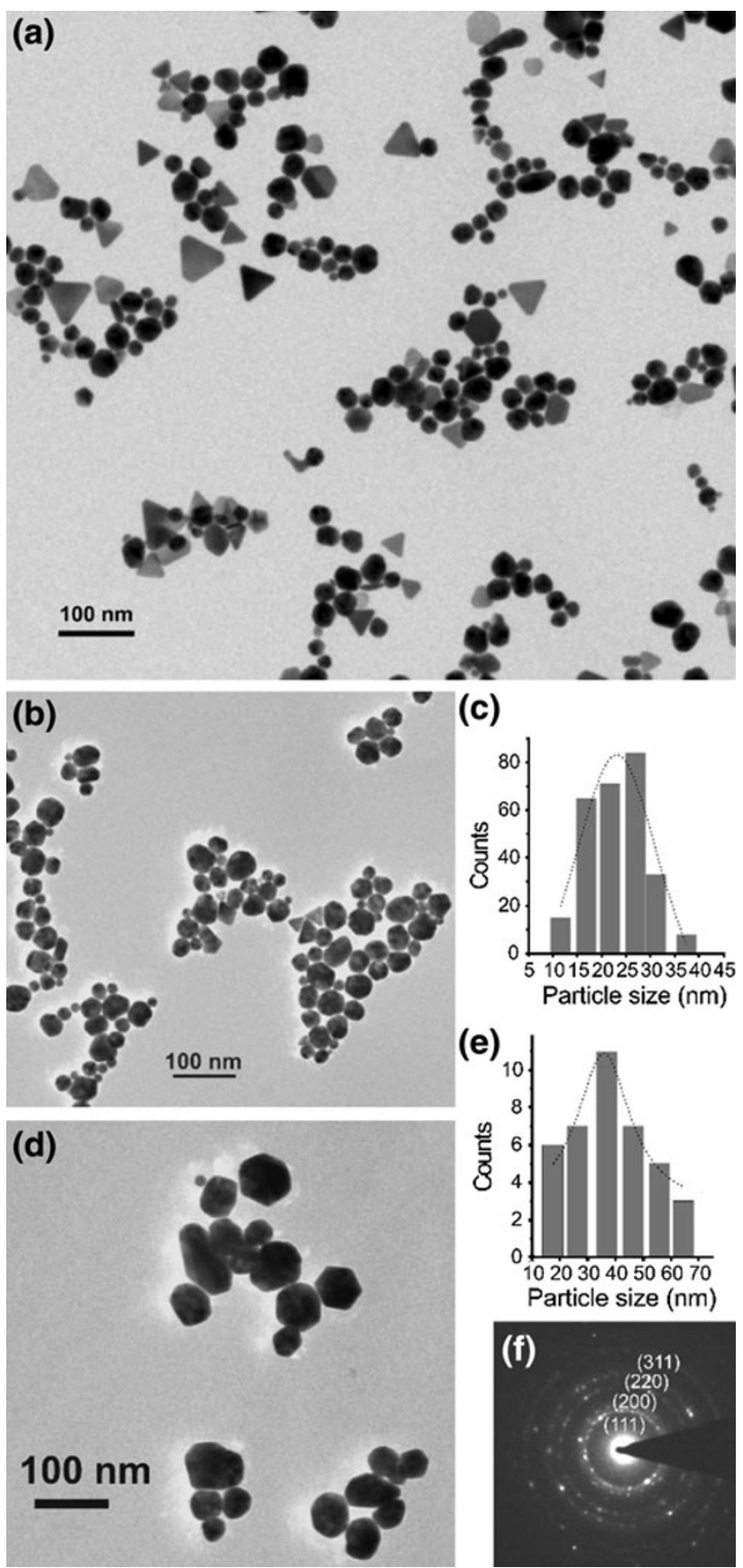

Fig. 2 a-c TEM Images of $\mathrm{Au}$ nanoparticles (concentration of extract $=2,400 \mathrm{mg} \mathrm{L}^{-1}$ ) and its histogram, d, e TEM images of $\mathrm{Au}$ nanoparticles (concentration of extract $=1,440 \mathrm{mg} \mathrm{L}^{-1}$ ) and its histogram, f SAED image

peak at $1,643 \mathrm{~cm}^{-1}$ is the characteristic of $\mathrm{C}=\mathrm{O}$ stretching of amide-I. The sharp absorption at $1,071 \mathrm{~cm}^{-1}$ belongs to $\mathrm{C}-\mathrm{O}-\mathrm{C}$ stretching vibration and the peak at $1,342 \mathrm{~cm}^{-1}$ corresponds to $-\mathrm{OH}$ in plane bending vibration. The presence of the biomass in the stabilized AuNPs was evident from the comparison of the FTIR spectra. 
Fig. 3 Mechanism of the formation and stabilization of AuNPs by polyphenolic compounds present in $P$. granatum juice:

(i) $P$. granatum juice containing polyphenolic compounds,

(ii) autoreduction and

stabilization by the

polyphenolic compounds

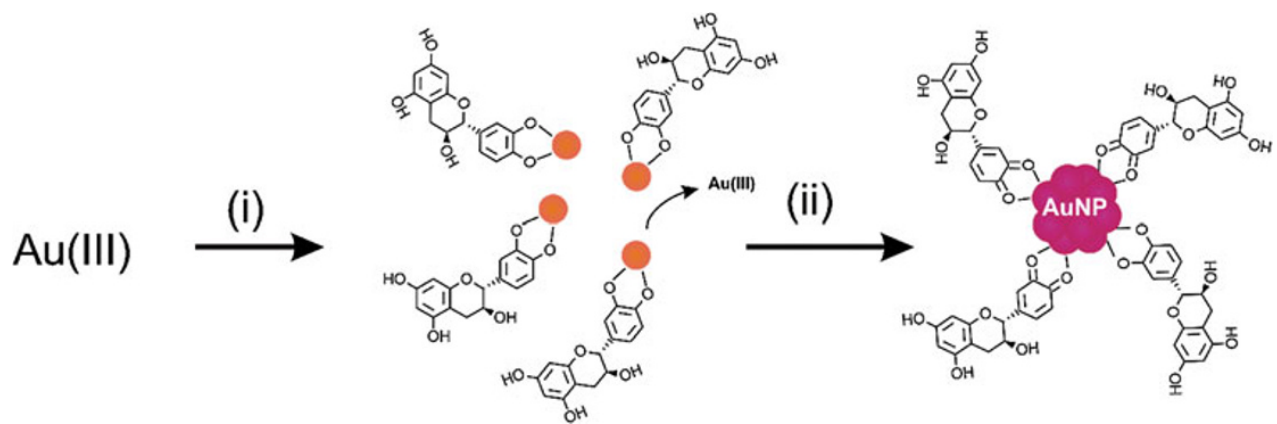

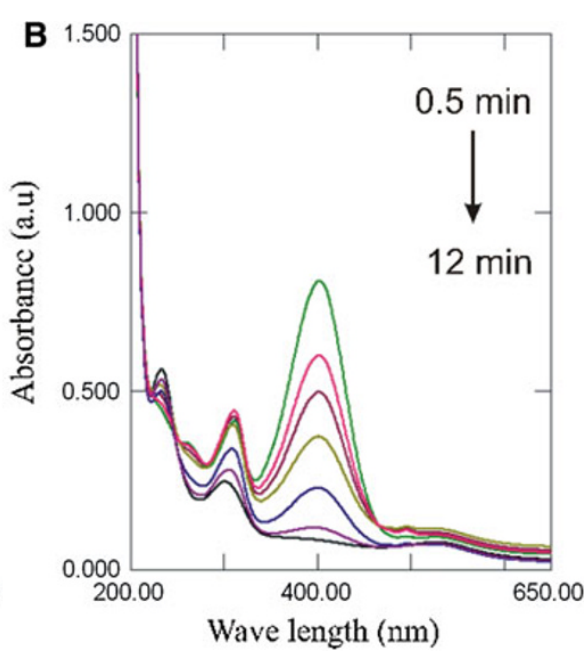

Fig. 4 A UV-visible spectrum of: (a) 4-nitrophenol $(0.05 \mathrm{mM}),(b)$ 4-nitrophenol in the presence of added sodium borohydride $(15 \mathrm{mM}),(c)$ after addition of AuNPs (20 min) catalyst in the reaction mixture. B Overlay of UV-visible spectra showing the progress of the reduction reaction at different time intervals

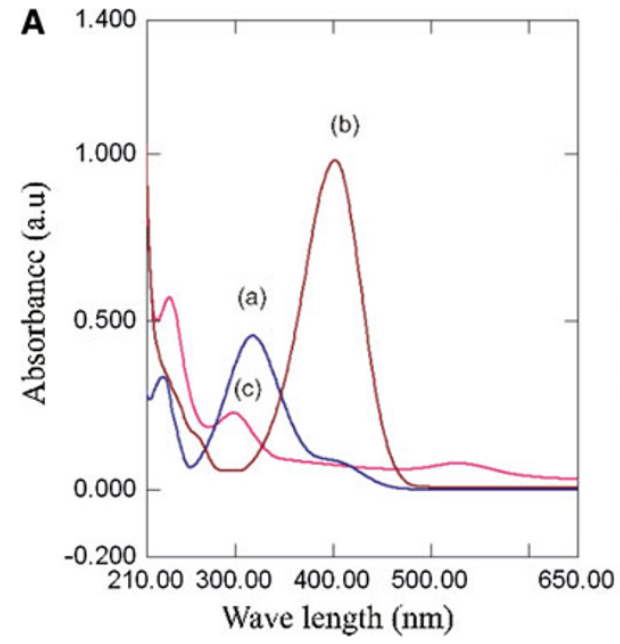

Reaction mechanism

A schematic representation of a possible mechanism for the synthesis and stabilization of AuNPs from the polyphenolic compounds present in P. granatum juice is shown in Fig. 3. A five-membered chelate ring is formed by the adjacent hydroxyl groups of the polyphenolic compounds. The chelated ortho-dihydroxy groups are then oxidized to quinones with concomitant reduction of $\mathrm{Au}^{3+}$ to $\mathrm{Au}^{0}$ because of the very high oxidation-reduction potential of $\mathrm{Au}^{3+}$. Collision of the neighboring $\mathrm{Au}^{0}$ atoms leads to the formation of AuNPs and the synthesized AuNPs are stabilized by polyphenolic compounds as well as the quinones (Huang et al. 2010; Prakash and Prakash 2011).

\section{Application of AuNPs in catalysis}

The reduction of 4-nitrophenol to 4-aminophenol by sodium borohydride is a thermodynamically favorable reaction $\left(\mathrm{E}_{0}\right.$ for 4-nitrophenol/4-aminophenol -0.76 and for $\left.\mathrm{H}_{3} \mathrm{BO}_{3} / \mathrm{BH}_{4}{ }^{-}-1.33 \mathrm{~V}\right)$. However, on treatment of an aqueous solution of 4-nitrophenol $(0.05 \mathrm{mM})$ with a freshly prepared aqueous solution of sodium borohydride (15 $\mathrm{mM})$, the absorption maxima shifted from 318.5 to
$401.5 \mathrm{~nm}$ due to the formation of 4-nitrophenolate ion (Fig. 4). No reduction of the nitro group to amino group took place on keeping the reaction mixture for several days due to a large kinetic barrier for the reduction reaction. Interestingly, on addition of $P$. granatum juice extract (480 $\left.\mathrm{mg} \mathrm{L}^{-1}\right)$ derived colloidal gold nanoparticle $(0.1 \mathrm{~mL})$ to the reaction mixture, lowering of the intensity of the absorption peak at $401.5 \mathrm{~nm}$ was observed with concomitant formation of a new peak at $298 \mathrm{~nm}$ indicating the formation of 4-aminophenol. Such catalysis by colloidal AuNPs in the reduction of 4-nitrophenol to 4-aminophenol by facilitating electron relay from the donor $\mathrm{BH}_{4}{ }^{-}$to the acceptor 4-nitrophenol is well known in the literature (Aromal et al. 2012). Complete disappearance of the $401.5 \mathrm{~nm}$ peak was observed within $12 \mathrm{~min}$ indicating completion of the reduction demonstrating the catalytic activity of the synthesized colloidal AuNPs. As the concentration of $\mathrm{BH}_{4}^{-}$was much larger than that of 4-nitrophenol, a pseudo-first-order rate constant for the reduction reaction could be assumed. Utilizing the UV-visible data, the catalytic rate constant $(\mathrm{k})$ was calculated to be 0.22 $\min ^{-1}$ (supporting information Table TS1 and Fig. S7). This rate constant value was comparable to the recently reported value with related systems (Gangula et al. 2011). 


\section{Conclusions}

A very mild method for the synthesis of colloidal AuNPs has been reported by using $P$. granatum juice, a well known multivitamin drink of great medicinal importance. The polyphenolic compounds present in the juice concentrate of $P$. granatum acts as an effective reducing agent for the reduction of $\mathrm{Au}(\mathrm{III})$ to $\mathrm{Au}(0)$ and the AuNPs of 23-36 nm size were formed without any additional capping agent or stabilizer. By increasing the concentration of the juice extract a gradual blue-shift of the surface plasmon band was observed indicating the formation of smallersized nanoparticles. Variation of size of the nanoparticles was also confirmed by HRTEM analysis. The synthesized colloidal AuNPs have also been utilized as a catalyst for the borohydride reduction of 4-nitrophenol. As P. granatum juice has tremendous medicinal significance, the results described here will be useful for its biomedical applications as well as nanoscience and nanotechnology.

Acknowledgments BGB thanks CSIR, New Delhi (02(0068)/12/ EMR-II) for the research grant. SSD thanks CSIR, New Delhi for the research fellowship.

Open Access This article is distributed under the terms of the Creative Commons Attribution License which permits any use, distribution, and reproduction in any medium, provided the original author(s) and the source are credited.

\section{References}

Anastas PT, Kirchhoff MM (2002) Origins, current status, and future challenges of green chemistry. Acc Chem Res 35:686-694

Aromal SA, Vidhu VK, Philip D (2012) Green synthesis of welldispersed gold nanoparticles using Macrotyloma uniflorum. Spectrochimica Acta A 85:99-104

Bag BG, Dash SS (2011) First self-assembly study of betulinic acid, a renewable nano-sized, 6-6-6-6-5 pentacyclic monohydroxy triterpenic acid. Nanoscale 3:4564-4566

Bag BG, Majumdar R (2012) Self-assembly of a renewable nano-sized triterpenoid 18ß-glycyrrhetinic acid. RSC Adv 2:8623-8626

Bag BG, Paul K (2012) Vesicular and fibrillar gels by self-assembly of nanosized oleanolic acid. Asian J Org Chem 1:150-154

Bag BG, Garai C, Majumdar R, Laguerre M (2012) Natural triterpenoids as renewable nanos. Struct Chem 23:393-398

Beeram SR, Zamborini FP (2010) Purification of gold nanoplates grown directly on surfaces for enhanced localized surface plasmon resonance biosensing. ACS Nano 4:3633-3646

Chandran SP, Chaudhary M, Pasricha R, Ahmad A, Sastry M (2006) Synthesis of gold nanotriangles and silver nanoparticles using Aloe vera plant extracts. Biotechnol Prog 22:577-583
Daniel MC, Astruc D (2004) Gold nanoparticles: assembly, supramolecular chemistry, quantum-size-related properties, and applications toward biology, catalysis, and nanotechnology. Chem Rev 104:293

Dykman L, Khlebtsov N (2012) Gold nanoparticles in biomedical applications: recent advances and perspectives. Chem Soc Rev 41:2256-2282

Esumi K, Suzuki A, Yamahira A, Torigoe K (2000) Role of poly(amidoamine) dendrimers for preparing nanoparticles of gold, platinum, and silver. Langmuir 16:2604-2608

Faraday M (1857) Experimental relations of gold (and other metals) to light. Philos Trans R Soc London 147:145-181

Ferrer M, Reina R, Rossell O, Seco M (1999) Anionic iron clusters as building blocks for the synthesis of higher nuclearity compounds containing 11- and 12-group elements. Coordin Chem Rev 193-5:619-642

Gangula A, Podila R, Ramakrishna M, Karanam L, Janardhana C, Rao AM (2011) Catalytic reduction of 4-nitrophenol using biogenic gold and silver nanoparticles derived from Breynia rhamnoides. Langmuir 27:15268-15274

Huang X, Wu H, Liao X, Shia B (2010) One-step, size-controlled synthesis of gold nanoparticles at room temperature using plant tannin. Green Chem 12:395-399

Jain PK, Huang X, El-Sayed IH, El-Sayed MA (2008) Noble metals on the nanoscale: optical and photothermal properties and some applications in imaging, sensing, biology, and medicine. Acc Chem Res 41:1578-1586

Kim YH, Nakano Y (2005) Adsorption mechanism of palladium by redox within condensed-tannin gel. Water Res 39:1324-1330

Kumari A, Yadava SK, Yadava SC (2010) Biodegradable polymeric nanoparticles based drug delivery systems. Colloids Surf B Biointerfaces 75:1-18

Majumdar R, Bag BG (2012) Terminalia arjuna bark extract mediated size controlled synthesis of polyshaped gold nanoparticles and its application in catalysis. Int $\mathbf{J}$ Res Chem Environ 2:338-342

Montes MO, Maoral A, Deepak FL, Parsons JG, Yacaman JM, Peralta-Videa JR, Gardea-Torresdey JL (2011) Anisotropic gold nanoparticles and gold plates biosynthesis using alfalfa extracts. J Nanopart Res 13:3113-3121

Murphy CJ, Gole AM, Stone JW, Sisco PN, Alkilany AM, Goldsmith EC, Baxter SC (2008) Gold nanoparticles in biology: beyond toxicity to cellular imaging. Acc Chem Res 41:1721-1730

Prakash CVS, Prakash I (2011) Bioactive chemical constituents from pomegranate (Punica granatum) juice, seed and peel-a review. Int J Res Chem Environ 1:1-18

Saha K, Agasti SS, Kim C, Li X, Rotello VM (2012) Gold nanoparticles in chemical and biological sensing. Chem Rev 112:2739

Shiv Shankar S, Rai A, Ahmad A, Sastry M (2004) Rapid synthesis of $\mathrm{Au}, \mathrm{Ag}$, and bimetallic Au core-Ag shell nanoparticles using Neem (Azadirachta indica) leaf broth. J Colloid Interface Sci 275(2):496

Zhang Y, Cui X, Shi F, Deng Y (2012) Nano-gold catalysis in fine chemical synthesis. Chem Rev 112:2467 